\title{
On the fundamental solutions of a discontinuous fractional boundary value problem
}

\author{
Ali Yakar and Zulfigar Akdogan*
}

\section{"Correspondence:}

zulfigar.akdogan@gop.edu.tr

Department of Mathematics,

Gaziosmanpasa University,

Tasliciftlik Campus, Tokat, 60250,

Turkey

\begin{abstract}
The main purpose of this study is to investigate a fractional discontinuous Sturm-Liouville problem with transmission conditions. We shall consider a fractional boundary value problem involving an operator with two parts. It is shown that the eigenvalues and corresponding eigenfunctions of the main problem coincide with the eigenvalues and corresponding eigenfunctions of the constructed operator in Hilbert spaces.
\end{abstract}

Keywords: fractional calculus; fractional Sturm-Liouville problem; fractional boundary conditions; fractional transmission condition

\section{Introduction}

Fractional calculus has been a well-known topic since it was initiated in the seventeenth century and studied by many great mathematicians of the time. The history of fractional calculus can be found in $[1,2]$. It has been shown that in many applications, fractional derivatives based models provide more accurate solutions for real processes of anomalous systems than the integer order derivatives based models do [3-6]. After realizing the success of fractional differential equations in modeling real world problems, much work has been done in this branch of mathematics in recent decades. For some recent contributions about fractional differential equations and fractional dynamic systems, see [7-13] and the references therein.

The studies of Sturm-Liouville (S-L) problems have attracted the attention of many mathematicians and physicists since they have many useful applications in branches of science, theoretical and applied mathematics. In particular, [14-19] contains many references to problems in physics and mechanics.

On the other hand, the general theory and methods of boundary value problems with continuous coefficients are highly developed, very little is known about a general character of similar problems with discontinuities. In view of demands of modern technology, engineering and physics, Sturm-Liouville type problems with transmission conditions have become a very important place of research in recent years. Discontinuous boundary value problems with transmission conditions and their applications to the corresponding initial-boundary value problems for parabolic equations have been investigated by Mukthrov et al. in [20-23]. Also, some problems with transmission conditions

(c) The Author(s) 2017. This article is distributed under the terms of the Creative Commons Attribution 4.0 International License (http://creativecommons.org/licenses/by/4.0/), which permits unrestricted use, distribution, and reproduction in any medium, provided you give appropriate credit to the original author(s) and the source, provide a link to the Creative Commons license, and indicate if changes were made. 
which arise in mechanics (thermal conduction problem for a thin laminated plate) were studied in the article [24]. Akdogan et al. and Demirci et al. [25-28] studied a SturmLiouville problem with discontinuities in the case when an eigenparameter appears not only in the differential equation but also in both the boundary and transmission conditions.

As can be seen in the references cited above, there have been a lot of research papers on Sturm-Liouville problems but the authors generally take into account the case where classical integer order derivatives exist. In recent years, some previous works involving fractional operators for S-L problems have been published. Riverouse et al. [29] use some fractional composition operators to propose a fractional approach to the ordinary SturmLiouville problem and investigate the eigenvalues and eigenfunctions associated to these operators. In [30], the authors consider a new conformable fractional derivative and apply a functional compression-expansion fixed point theorem to prove the existence of a positive solution for fractional boundary value problem with S-L boundary conditions. In [31], utilizing the Legendre integral transform, the authors demonstrate some applications of their results by solving both fractional ordinary and partial differential equations. Zayernouri and Karniadakis [32] investigated two classes of fractional Sturm-Liouville eigenvalue problems on a compact interval $[a, b]$ in more detail. They both obtained some explicit forms for the eigensolutions of these problems and derived some useful spectral properties of the obtained eigensolutions. In [33], Klimek et al. apply the methods of fractional variational analysis and prove the existence of a countable set of orthogonal solutions and corresponding eigenvalues for a regular fractional Sturm-Liouville problem.

The main aim of this paper is to extend some results of fractional Sturm-Liouville problems to the case of discontinuous fractional Sturm-Liouville problems. Namely, we deal with a discontinuous fractional Sturm-Liouville problem with transmission conditions. To do so, we define an operator $A$ in the Hilbert space $L_{2}[-1,1]$, the eigenvalues and corresponding eigenfunctions of which coincide with the eigenvalues and corresponding eigenfunctions of the boundary value problem respectively. Then, we establish the characteristic function and prove that the eigenvalues of the considered problem coincide with the roots of this characteristic function.

The paper is organized as follows: In Section 2, some of the basic properties of the Riemann-Liouville and Caputo fractional derivatives are given, and we also prove a useful lemma. Section 3 presents the construction of a discontinuous fractional S-L problem. We introduce the operator theoretical form in the Hilbert space $L_{2}[-1,1]$ and discuss the characteristic function in the subsequent sections.

\section{Some auxiliary definitions and results}

In this section, we shall recall some basic definitions and facts which are necessary for the development of the paper (see also $[1,2]$ ).

Definition 1 (Left and right Riemann-Liouville fractional integrals) Let $[a, b] \subset \Re$, $\operatorname{Re}(\alpha)>0$ and $f \in L_{1}[a, b]$. Then the left and right Riemann-Liouville fractional integrals 
$I_{a+}^{\alpha}$ and $I_{b^{-}}^{\alpha}$ of order $\alpha$ are given by

$$
\begin{aligned}
& I_{a+}^{\alpha} f(x)=\frac{1}{\Gamma(\alpha)} \int_{a}^{x} \frac{f(t) d t}{(x-t)^{1-\alpha}}, \quad x \in(a, b], \\
& I_{b-}^{\alpha} f(x)=\frac{1}{\Gamma(\alpha)} \int_{x}^{b} \frac{f(t) d t}{(t-x)^{1-\alpha}}, \quad x \in[a, b) .
\end{aligned}
$$

Definition 2 (Left and right Riemann-Liouville (R-L) fractional derivatives) Let $[a, b] \subset$ $\Re, \operatorname{Re}(\alpha) \in(0,1)$ and $f \in L_{1}[a, b]$. The left R-L fractional derivative of order $\alpha$ of function $f$, denoted by $D_{a+}^{\alpha} f$, is defined as

$$
\forall x \in(a, b], \quad D_{a+}^{\alpha} f(x):=D I_{a+}^{1-\alpha} f(x) .
$$

Similarly, the right Riemann-Liouville fractional derivative of order $\alpha$ of function $f$, denoted by $D_{b-}^{\alpha} f$, is

$$
\forall x \in[a, b), \quad D_{b^{-}}^{\alpha} f(x):=-D I_{b^{-}}^{1-\alpha} f(x),
$$

where $D=\frac{d}{d x}$ is the usual differential operator.

Definition 3 (Left and right Caputo fractional derivatives) Let $[a, b] \subset \Re, \operatorname{Re}(\alpha) \in(0,1)$ and $f \in L_{1}[a, b]$. The left and right Caputo fractional derivatives of order $\alpha$ are

$$
\forall x \in(a, b], \quad{ }^{c} D_{a+}^{\alpha} f(x):=I_{a+}^{1-\alpha} D f(x)
$$

and

$$
\forall x \in[a, b), \quad{ }^{c} D_{b^{-}}^{\alpha} f(x):=-I_{b^{-}}^{1-\alpha} D f(x)
$$

respectively.

Property 1 The following property shows that the Riemann-Liouville derivative is the left inverse of the Riemann-Liouville integral, but we cannot claim that it is the right inverse.

$$
\begin{aligned}
& D_{a+}^{\alpha} I_{a+}^{\alpha} f(x)=f(x), \\
& D_{b^{-}}^{\alpha} I_{b^{-}}^{\alpha} f(x)=f(x)
\end{aligned}
$$

and

$$
\begin{aligned}
& I_{a+}^{\alpha} D_{a+}^{\alpha} f(x)=f(x)-\frac{(x-a)^{\alpha-1}}{\Gamma(\alpha)} I_{a+}^{1-\alpha} f(a), \\
& I_{b^{-}}^{\alpha} D_{b^{-}}^{\alpha} f(x)=f(x)-\frac{(b-x)^{\alpha-1}}{\Gamma(\alpha)} I_{a+}^{1-\alpha} f(b),
\end{aligned}
$$

where $\alpha \in(0,1)$. 
Property 2 For certain classes of functions, the Caputo fractional derivatives are the inverse operators of the Riemann-Liouville fractional integrals.

$$
\begin{aligned}
& { }^{c} D_{a+}^{\alpha} I_{a+}^{\alpha} f(x)=f(x), \\
& { }^{c} D_{b^{-}}^{\alpha} I_{b^{-}}^{\alpha} f(x)=f(x)
\end{aligned}
$$

and

$$
\begin{aligned}
I_{a+}^{\alpha}{ }^{c} D_{a+}^{\alpha} f(x) & =f(x)-f(a), \\
I_{b^{-}}^{\alpha}{ }^{c} D_{b^{-}}^{\alpha} f(x) & =f(x)-f(b) .
\end{aligned}
$$

In classical calculus, the integration by parts formula relates the integral of a product of functions to the integral of their derivative and antiderivative. As we can see below, this formula also works for fractional derivatives; however, it changes the type of differentiation: left Riemann-Liouville fractional derivatives are transformed to right Caputo fractional derivatives. For more detail see, for example, [31].

Property 3 Assume that $0<\alpha<1, f \in A C[a, b]$ and $g \in L^{p}(a, b)(1 \leq p \leq \infty)$. Then the following integration by parts formula holds:

$$
\int_{a}^{b} f(x) D_{a+}^{\alpha} g(x) d x=\int_{a}^{b} g(x)^{c} D_{b^{-}}^{\alpha} f(x) d x+\left.f(x) I_{a+}^{1-\alpha} g(x)\right|_{x=a} ^{x=b} .
$$

Now, we state and prove the following lemma which is going to be used in the next section.

Lemma 1 Let $f \in L_{2}(a, b)$ and $\alpha \in(0,1)$, then

1. $I_{a^{+}}^{\alpha}{ }^{c} D_{b}^{\alpha} f(x)=M_{g}(x)+(-1)^{\alpha}(f(x)-f(b))$,

2. $I_{a^{+}}^{\alpha} D_{b-}^{\alpha} f(x)=(-1)^{\alpha-1} I_{a^{+}}^{\alpha} N_{f}(x)+(-1)^{\alpha}(f(x)-f(a))$,

where $M_{g}(x)=\frac{1}{\Gamma(\alpha)} \int_{a}^{b}(x-t)^{\alpha-1} g(t) d t, N_{f}(x)=\frac{1}{\Gamma(1-\alpha)} \int_{a}^{b}(x-t)^{-\alpha} f^{\prime}(t) d t$ and $g(x)={ }^{c} D_{b}^{\alpha} f(x)$.

Proof In view of Definition 1, we have

$$
\begin{aligned}
M_{g}(x) & =\frac{1}{\Gamma(\alpha)} \int_{a}^{x}(x-t)^{\alpha-1} g(t) d t+\frac{1}{\Gamma(\alpha)} \int_{x}^{b}(x-t)^{\alpha-1} g(t) d t \\
& =I_{a^{+}}^{\alpha} g(x)+(-1)^{\alpha-1} I_{b^{-}}^{\alpha} g(x) .
\end{aligned}
$$

Then it leads to

$$
I_{a^{+}}^{\alpha} g(x)=M_{g}(x)+(-1)^{\alpha} I_{b^{-}}^{\alpha} g(x)
$$

To prove (2), by Definition 3, we obtain

$$
\begin{aligned}
N_{f}(x) & =\frac{1}{\Gamma(1-\alpha)} \int_{a}^{x}(x-t)^{-\alpha} f^{\prime}(t) d t+\frac{1}{\Gamma(1-\alpha)} \int_{x}^{b}(x-t)^{-\alpha} f^{\prime}(t) d t \\
& ={ }^{c} D_{a^{+}}^{\alpha} f(x)+\frac{1}{\Gamma(1-\alpha)} \int_{x}^{b}(t-x)^{-\alpha}(-1)^{-\alpha+1}\left(-f^{\prime}\right)(t) d t \\
& ={ }^{c} D_{a^{+}}^{\alpha} f(x)+(-1)^{1-\alpha c} D_{b^{-}}^{\alpha} f(x),
\end{aligned}
$$


which gives

$$
{ }^{c} D_{b^{-}}^{\alpha} f(x)=\left(N_{f}(x)-{ }^{c} D_{a^{+}}^{\alpha} f(x)\right)(-1)^{\alpha-1} .
$$

By applying the fractional operator $I_{a^{+}}^{\alpha}$ to both sides, we get

$$
\begin{aligned}
I_{a^{+}}^{\alpha c} D_{b^{-}}^{\alpha} f(x) & =I_{a^{+}}^{\alpha}\left(N_{f}(x)-{ }^{c} D_{a^{+}}^{\alpha} f(x)\right)(-1)^{\alpha-1} \\
& =(-1)^{\alpha-1} I_{a^{+}}^{\alpha} N_{f}(x)+(-1)^{\alpha} I_{a^{+}}^{\alpha c} D_{a^{+}}^{\alpha} f(x) \\
& =(-1)^{\alpha-1} I_{a^{+}}^{\alpha} N_{f}(x)+(-1)^{\alpha}(f(x)-f(a)),
\end{aligned}
$$

which completes the proof.

\section{Fractional Sturm-Liouville problem with transmission conditions}

Let the operator $\mathfrak{L}_{\alpha, x}$ be defined as

$$
\begin{aligned}
& \mathfrak{L}_{\alpha, x}:= \begin{cases}{ }^{c} D_{0^{-}}^{\alpha} p(x) D_{-1^{+}}^{\alpha}+q(x), & x \in[-1,0) ; \\
{ }^{c} D_{1^{-}}^{\alpha} p(x) D_{0^{+}}^{\alpha}+q(x), & x \in(0,1] .\end{cases} \\
& \mathfrak{L}_{\alpha, x} u+\lambda u=0
\end{aligned}
$$

on $x \in[-1,0) \cup(0,1]$ with fractional boundary conditions

$$
\begin{aligned}
& L_{1}(u):=c_{1} I_{-1^{+}}^{1-\alpha} u(-1)+c_{2} D_{-1^{+}}^{\alpha} u(-1)=0, \\
& L_{2}(u):=d_{1} I_{0^{+}}^{1-\alpha} u(1)+d_{2} D_{0^{+}}^{\alpha} u(1)=0
\end{aligned}
$$

and fractional transmission conditions at the inner point $x=0$

$$
\begin{aligned}
& L_{3}(u):=h_{1} I_{-1^{+}}^{1-\alpha} u(-0)+I_{0^{+}}^{1-\alpha} u(+0)=0, \\
& L_{4}(u):=D_{-1^{+}}^{\alpha} u(-0)+h_{2} D_{0^{+}}^{\alpha} u(+0)=0,
\end{aligned}
$$

where $\frac{1}{2}<\alpha \leq 1, \lambda$ is complex eigenparameter;

$$
p(x)= \begin{cases}p_{1}, & x \in[-1,0) ; \\ p_{2}, & x \in(0,1] .\end{cases}
$$

$q(x)$ is real-valued and continuous in both $[-1,0)$ and $(0,1]$, it also has finite limits $q( \pm 0):=$ $\lim _{x \rightarrow \pm 0} q(x), c_{1}^{2}+c_{2}^{2} \neq 0, d_{1}^{2}+d_{2}^{2} \neq 0$, and $p_{1}, p_{2}, h_{1}, h_{2}$ are positive real numbers.

\section{The operator formulation of the problem}

Let us consider the inner-product in the Hilbert space $L_{2}(-1,1)$ as follows:

$$
\langle f, g\rangle=\frac{h_{1}}{p_{1}} \int_{-1}^{0} f(x) \overline{g(x)} d x+\frac{h_{2}}{p_{2}} \int_{0}^{1} f(x) \overline{g(x)} d x
$$


where $F:=f(x), G:=g(x) \in L_{2}(-1,1)$. In this Hilbert space we define the operator $A$ with domain

$$
D(A):=\left\{\begin{array}{l}
f: f(x) \text { and } D^{\alpha} f(x),{ }^{c} D^{\alpha} f(x) \text { are absolutely continuous } \\
\text { on }[-1,0) \cup(0,1], \text { and } f(0 \pm), D^{\alpha} f(0 \pm), I^{1-\alpha} f(0 \pm) \text { have } \\
\text { finite limits, } L_{i} f=0, i=1,2,3,4 .
\end{array}\right\}
$$

and action law

$$
\text { Af }:=\mathfrak{L}_{\alpha, x} f \text {. }
$$

Thus, problem (2)-(6) can be written in the operator form as

$$
A u=\lambda u .
$$

Note that by eigenvalues and eigenfunctions of problem (2)-(6) we mean eigenvalues and eigenelements of the operator $A$, respectively.

Theorem 1 The linear operator A is symmetric.

Proof For each $f, g \in \operatorname{Dom}(A)$, using (7) we write

$$
\begin{aligned}
& \langle A f, g\rangle=\frac{h_{1}}{p_{1}} \int_{-1}^{0} A f(x) \overline{g(x)} d x+\frac{h_{2}}{p_{2}} \int_{0}^{1} A f(x) \overline{g(x)} d x \\
& =\frac{h_{1}}{p_{1}} \int_{-1}^{0}\left({ }^{c} D_{0^{-}}^{\alpha} p_{1} D_{-1^{+}}^{\alpha} f(x)\right) \overline{g(x)} d x+\frac{h_{1}}{p_{1}} \int_{-1}^{0} q(x) f(x) \overline{g(x)} d x
\end{aligned}
$$

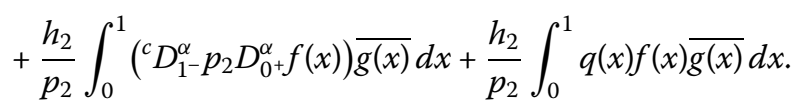

By applying Property 3, we get

$$
\begin{aligned}
\langle A f, g\rangle= & h_{1}\left\{\int_{-1}^{0} f(x)^{c} D_{0^{-}}^{\alpha} D_{-1^{+}}^{\alpha} \overline{g(x)} d x+\left.D_{-1^{+}}^{\alpha} \overline{g(x)} I_{-1^{+}}^{1-\alpha} f(x)\right|_{-1} ^{0}-\left.D_{-1^{+}}^{\alpha} f(x) I_{-1^{+}}^{1-\alpha} \overline{g(x)}\right|_{-1} ^{0}\right\} \\
& +h_{2}\left\{\int_{0}^{1} f(x)^{c} D_{1^{-}}^{\alpha} D_{0^{+}}^{\alpha} \overline{g(x)} d x+\left.D_{0^{+}}^{\alpha} \overline{g(x)} I_{0^{+}}^{1-\alpha} f(x)\right|_{0} ^{1}-\left.D_{0^{+}}^{\alpha} f(x) I_{0^{+}}^{1-\alpha} \overline{g(x)}\right|_{0} ^{1}\right\} \\
& +\frac{h_{1}}{p_{1}} \int_{-1}^{0} q(x) f(x) \overline{g(x)} d x+\frac{h_{2}}{p_{2}} \int_{0}^{1} q(x) f(x) \overline{g(x)} d x \\
= & \langle f, A g\rangle+h_{1}\left\{\left.D_{-1^{+}}^{\alpha} \overline{g(x)} I_{-1^{+}}^{1-\alpha} f(x)\right|_{-1} ^{0}-\left.D_{-1^{+}}^{\alpha} f(x) I_{-1^{+}}^{1-\alpha} \overline{g(x)}\right|_{-1} ^{0}\right\} \\
& +h_{2}\left\{\left.D_{0^{+}}^{\alpha} \overline{g(x)} I_{0^{+}}^{1-\alpha} f(x)\right|_{0} ^{1}-\left.D_{0^{+}}^{\alpha} f(x) I_{0^{+}}^{1-\alpha} \overline{g(x)}\right|_{0^{1}} ^{1}\right\} .
\end{aligned}
$$

By considering the fractional transmission conditions (5)-(6), we have

$$
\langle A f, g\rangle=\langle f, A g\rangle
$$

that the operator $A$ is symmetric. 
Corollary 1 All eigenvalues of problem (2)-(6) are real.

Corollary 2 Let $\lambda_{1}$ and $\lambda_{2}$ be two different eigenvalues of problem (2)-(6). Then the corresponding eigenfunctions $f$ and $g$ of this problem satisfy the following equality:

$$
\frac{h_{1}}{p_{1}} \int_{-1}^{0} A f(x) \overline{g(x)} d x+\frac{h_{2}}{p_{2}} \int_{0}^{1} A f(x) \overline{g(x)} d x=0
$$

As a consequence, the eigenfunctions of problem (2)-(6) corresponding to the different eigenvalues are orthogonal to the inner product (7) in the Hilbert space $L_{2}(-1,1)$.

Naturally, we can now assume that all eigenfunctions of problem (2)-(6) are realvalued.

Lemma 2 The equivalent integral form of equation (2) with fractional conditions (5)-(6) is given as

$$
u(x)=u_{0}(x)+\frac{1}{p_{2} \Gamma(2 \alpha)} \int_{0}^{x}\left[N_{u}(y)+(-1)^{1-\alpha}(x-y)^{2 \alpha-1}(\lambda+q(y)) u(y)\right] d y,
$$

where $u_{0}(x)=\frac{x^{\alpha-1}}{\Gamma(\alpha)}\left(-h_{1} I_{-1^{+}}^{1-\alpha} u(-0)\right)+I_{0^{+}}^{\alpha}\left(-\frac{1}{h_{2}} D_{-1^{+}}^{\alpha} u(-0)\right)$.

Proof Let us consider equation (2)

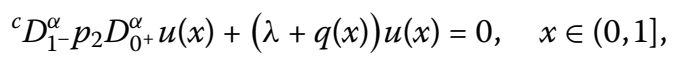

integral operators $I_{0^{+}}^{\alpha}$ acting on this equation and by Lemma 1, we obtain

$$
I_{0^{+}}^{\alpha}\left({ }^{c} D_{1^{-}}^{\alpha} p_{2} D_{0^{+}}^{\alpha} u(x)\right)+I_{0^{+}}^{\alpha}(\lambda+q(x)) u(x)=0
$$

and

$$
p_{2} D_{0^{+}}^{\alpha} u(x)=I_{0^{+}}^{\alpha} N_{u}(x)+p_{2} D_{0^{+}}^{\alpha} u(+0)+(-1)^{1-\alpha} I_{0^{+}}^{\alpha}(\lambda+q(x)) u(x) .
$$

Applying $I_{0^{+}}^{\alpha}$ on both sides of (11) and using conditions (5)-(6), we find

$$
\begin{aligned}
u(x)= & \frac{x^{\alpha-1}}{\Gamma(\alpha)}\left(-h_{1} I_{-1^{+}}^{1-\alpha} u(-0)\right)+I_{0^{+}}^{\alpha}\left(-\frac{1}{h_{2}} D_{-1^{+}}^{\alpha} u(-0)\right)+\frac{1}{p_{2}} I_{0^{+}}^{2 \alpha} N_{u}(x) \\
& +\frac{(-1)^{1-\alpha}}{p_{2}} I_{0^{+}}^{2 \alpha}(\lambda+q(y)) u(x) .
\end{aligned}
$$

Then we reach

$$
u(x)=u_{0}(x)+\frac{1}{p_{2}} I_{0^{+}}^{2 \alpha}\left[N_{u}(x)+(-1)^{1-\alpha}(\lambda+q(x)) u(x)\right]
$$

which completes the proof. 
We next define $u_{m}(x, \lambda)$ to construct the successive approximations

$$
u_{m}(x, \lambda)=u_{0}(x, \lambda)+\frac{1}{p_{2} \Gamma(2 \alpha)} \int_{0}^{x}(x-y)^{2 \alpha-1}\left[N_{u_{m-1}}(y)+(-1)^{1-\alpha}(\lambda+q(y)) u_{m-1}(y)\right] d y
$$

Remark 1 The corresponding classical Sturm-Liouville problem with integer orders is covered by the case $\alpha=1$.

Lemma 3 Let $Q:=\max _{x \in(0,1]}|q(x)|, P_{R}:=\max _{|\lambda| \leq \mathbb{R}} P(\lambda)$ and $P(\lambda):=\max _{x \in(0,1]}\left|u_{0}(x, \lambda)\right|$, $k_{\alpha}:=1 /[(2-\alpha) \Gamma(1-\alpha)]$. Then the following estimate

$$
\left\|u_{m}(x, \lambda)-u_{m-1}(x, \lambda)\right\| \leq P_{R}\left\{\frac{|\lambda|+2 k_{\alpha}+Q}{p_{2} \Gamma(2 \alpha+1)}\right\}^{m}
$$

holds for all $m$.

Proof Let us apply the mathematical induction for $m$. In what follows, for convenience we shall use the notation $K=1 / \Gamma(2 \alpha+1)$.

For $m=1$, we have

$$
\left\|u_{1}(x, \lambda)-u_{0}(x, \lambda)\right\|=\left\|\frac{1}{p_{2}} I_{0^{+}}^{2 \alpha}\left(N_{u_{0}}(x)+(-1)^{1-\alpha}(\lambda+q(x)) u_{0}(x, \lambda)\right)\right\| .
$$

By using Lemma 2.1 in [2], we have

$$
\begin{aligned}
\left\|u_{1}(x, \lambda)-u_{0}(x, \lambda)\right\| & \leq \frac{1}{p_{2}} K\left\|N_{u_{0}}(x)+(-1)^{1-\alpha}(\lambda+q(x)) u_{0}(x, \lambda)\right\| \\
& \leq \frac{1}{p_{2}} K\left[\left\|N_{u_{0}}(x)\right\|+\left\|(\lambda+q(x)) u_{0}(x, \lambda)\right\|\right] .
\end{aligned}
$$

By using Corollary 2.3 in [2], we have

$$
\begin{aligned}
\left\|u_{1}(x, \lambda)-u_{0}(x, \lambda)\right\| & \leq \frac{K}{p_{2}}\left[2 k_{\alpha}\left\|u_{0}(x, \lambda)\right\|+(|\lambda|+Q)\left\|u_{0}(x, \lambda)\right\|\right] \\
& \leq \frac{K P_{R}}{p_{2}}\left(2 k_{\alpha}+|\lambda|+Q\right) .
\end{aligned}
$$

Suppose that (13) holds for $m$ - 1, i.e.,

$$
\left\|u_{m-1}(x, \lambda)-u_{m-2}(x, \lambda)\right\| \leq P_{R}\left\{\frac{K}{p_{2}}\left(|\lambda|+2 k_{\alpha}+Q\right)\right\}^{m-1}
$$

Then we have

$$
\begin{aligned}
& \left\|u_{m}(x, \lambda)-u_{m-1}(x, \lambda)\right\| \\
& =\left\|\frac{1}{p_{2}} I_{0^{+}}^{2 \alpha}\left[N_{u_{m-1}-u_{m-2}}(x)+(-1)^{\alpha}(\lambda+q(x))\left(u_{m-1}(x, \lambda)-u_{m-2}(x, \lambda)\right)\right]\right\| \\
& \leq \frac{K}{p_{2}}\left[\left\|N_{u_{m-1}-u_{m-2}}(x)\right\|+\left\|(\lambda+q(x))\left(u_{m-1}(x, \lambda)-u_{m-2}(x, \lambda)\right)\right\|\right]
\end{aligned}
$$




$$
\begin{aligned}
& \leq \frac{K}{p_{2}}\left[2 k_{\alpha}\left\|\left(u_{m-1}(x, \lambda)-u_{m-2}(x, \lambda)\right)\right\|+(|\lambda|+Q)\left\|\left(u_{m-1}(x, \lambda)-u_{m-2}(x, \lambda)\right)\right\|\right] \\
& =\frac{K}{p_{2}}\left(2 k_{\alpha}+|\lambda|+Q\right)\left\|\left(u_{m-1}(x, \lambda)-u_{m-2}(x, \lambda)\right)\right\| \\
& \leq P_{R}\left\{\frac{K}{p_{2}}\left(|\lambda|+2 k_{\alpha}+Q\right)\right\}^{m} .
\end{aligned}
$$

The proof is completed.

Lemma 4 The following IVP

$$
\begin{aligned}
& { }^{c} D_{0^{-}}^{\alpha} p_{1} D_{-1^{+}}^{\alpha} u(x)+(q(x)+\lambda) u(x)=0, \quad x \in[-1,0], \\
& I_{-1^{+}}^{1-\alpha} u(-1)=c_{2}, \\
& D_{-1^{+}}^{\alpha} u(-1)=-c_{1}
\end{aligned}
$$

has a unique solution on $[-1,0]$ provided that

$$
\frac{K}{p_{1}}\left(|\lambda|+2 k_{\alpha}+Q\right)<1
$$

Proof If we use a similar way in Lemma 2, we get a corresponding integral equation of the problem as follows:

$$
u(x)=u_{0}(x)+\frac{1}{p_{1}} I_{-1^{+}}^{2 \alpha}\left[N_{u}(x)+(-1)^{1-\alpha}(\lambda+q(x)) u(x)\right]
$$

where $u_{0}(x)=\frac{(x+1)^{\alpha}}{\Gamma(1+\alpha)}\left(-c_{1}\right)+\frac{(x+1)^{\alpha-1}}{\Gamma(\alpha)} c_{2}$.

Let us construct the integral equation by

$$
\phi=T \phi,
$$

where the mapping $T$ is defined as

$$
T f=u_{0}+\frac{1}{p_{1}} I_{-1^{+}}^{2 \alpha}\left[N_{f}+(-1)^{1-\alpha}(\lambda+q) f\right],
$$

then we have

$$
\|T f-T g\|=\left\|\frac{1}{p_{1}} I_{-1^{+}}^{2 \alpha}\left[\left(N_{f}-N_{g}\right)+(-1)^{1-\alpha}(\lambda+q)(f-g)\right]\right\| .
$$

By applying Lemma 2.1 in [2], we get

$$
\begin{aligned}
\|T f-T g\| & \leq \frac{K}{p_{1}}\left\|\left(N_{f}-N_{g}\right)+(-1)^{1-\alpha}(\lambda+q)(f-g)\right\| \\
& \leq \frac{K}{p_{1}}\left\|\left(N_{f}-N_{g}\right)\right\|+\|(\lambda+q)(f-g)\| .
\end{aligned}
$$


By relation (1) we have

$$
N_{f}-N_{g}={ }^{c} D_{-1^{+}}^{\alpha}(f-g)+(-1)^{1-\alpha c} D_{1^{-}}^{\alpha}(f-g),
$$

then

$$
\begin{aligned}
\left\|N_{f}-N_{g}\right\| & \leq k_{\alpha}\|f-g\|+k_{\alpha}\|f-g\| \\
& =2 k_{\alpha}\|f-g\|,
\end{aligned}
$$

where we have used Corollary 2.3 in [2]. If we substitute the last inequality into (20), we find

$$
\|T f-T g\| \leq \frac{K}{p_{1}}\left(|\lambda|+2 k_{\alpha}+Q\right)\|f-g\| .
$$

By condition (17), the mapping $T$ is a contraction on the space $\langle C[-1,0],\|\cdot\|\rangle$. Consequently, there exists a unique solution of equation (19). The proof is complete.

Theorem 2 For any $\lambda \in \mathbb{C}$ satisfying $K p_{i}^{-1}\left(|\lambda|+2 k_{\alpha}+Q\right)<1(i=1,2)$, the differential equation (2) has a unique solution which satisfies fractional boundary condition (3) and fractional transmission conditions (5)-(6).

Proof Consider the following problem for each $\lambda \in \mathbb{C}$ :

$$
\begin{aligned}
& \mathfrak{L}_{\alpha, x} u(x)+\lambda u(x)=0, \quad x \in[-1,0), \\
& { }^{c} D_{0^{-}}^{\alpha} p_{1} D_{-1^{+}}^{\alpha} u(x)+(q(x)+\lambda) u(x)=0, \quad x \in[-1,0), \\
& I_{-1^{+}}^{1-\alpha} u(-1)=c_{2}, \\
& D_{-1^{+}}^{\alpha} u(-1)=-c_{1} .
\end{aligned}
$$

By considering Lemma 4, the initial value problem has a unique solution $\phi_{1}(x, \lambda)$.

Next, take into account the differential equation

$$
\begin{aligned}
& \mathfrak{L}_{\alpha, x} u(x)+\lambda u(x)=0, \quad x \in(0,1], \\
& { }^{c} D_{1^{-}}^{\alpha} p_{2} D_{0^{+}}^{\alpha} u(x)+(q(x)+\lambda) u(x)=0, \quad x \in(0,1], \\
& I_{0^{+}}^{1-\alpha} u(+0)=-h_{1} I_{-1^{+}}^{1-\alpha} \phi_{1}(-0), \\
& D_{0^{+}}^{\alpha} u(+0)=-\frac{1}{h_{2}} D_{-1^{+}}^{\alpha} \phi_{1}(-0) .
\end{aligned}
$$

We establish the sequence $\left\{u_{n}(x, \lambda)\right\}$ for $x \in(0,1]$ and $n=1,2, \ldots$ such that

$$
\begin{aligned}
u_{n}(x, \lambda)= & u_{0}(x, \lambda) \\
& +\frac{1}{p_{2} \Gamma(2 \alpha)} \int_{0}^{x}(x-y)^{2 \alpha-1}\left[N_{u_{n-1}}(y)+(-1)^{1-\alpha}(\lambda+q(y))\right] u_{n-1}(y, \lambda) d y,
\end{aligned}
$$


where

$$
u_{0}(x, \lambda)=I_{-1^{+}}^{\alpha}\left(k D_{-1^{+}}^{\alpha} \phi_{1}(-0, \lambda)\right), \quad x \in(0,1] .
$$

Obviously, each of the functions $u_{n}(x, \lambda)$ is an entire function of $\lambda$ for each $x \in(0,1]$.

Now let us consider the series

$$
u^{*}(x, \lambda)=\lim _{n \rightarrow \infty}\left(u_{n}(x, \lambda)-u_{0}(x, \lambda)\right)=\sum_{j=1}^{\infty}\left(u_{j}(x, \lambda)-u_{j-1}(x, \lambda)\right) .
$$

According to estimate (13) in Lemma 3 , for $0<x \leq 1$, the absolute value of its terms is less than the corresponding terms of the convergent numeric series

$$
P_{R} \sum_{j=1}^{\infty}\left\{\frac{K}{p_{2}}\left(|\lambda|+2 k_{\alpha}+Q\right)\right\}^{j}
$$

Hence, series (31) converges uniformly. Obviously, each term $\left(u_{j}(x, \lambda)-u_{j-1}(x, \lambda)\right)$ of series (31) is continuous on $x \in(0,1]$. Therefore, the sum of series (31) is continuous on $x \in(0,1]$ and

$$
\phi_{2}(x, \lambda)=\lim _{n \rightarrow \infty} u_{n}(x, \lambda)=u_{0}(x, \lambda)+u^{*}(x, \lambda)
$$

is continuous on $x \in(0,1]$.

The uniform convergency of the sequence $u_{n}(x, \lambda)$ allows us to take $n \rightarrow \infty$ in the relation (29). This gives equations (12) showing that $\phi_{2}(x, \lambda)$, the limit function of the process defined by (30) and (29), is the solution of (12). Furthermore, it is trivial that $\phi_{2}(x, \lambda)$ satisfies the initial conditions (27)-(28). Finally, the function $\phi(x, \lambda)$ given by

$$
\phi(x, \lambda)= \begin{cases}\phi_{1}(x, \lambda), & x \in[-1,0) \\ \phi_{2}(x, \lambda), & x \in(0,1]\end{cases}
$$

satisfies the differential equation (2), fractional boundary condition (3) and fractional transmission conditions (5) and (6).

In a similar manner, we can prove the following theorem.

Theorem 3 For any $\lambda \in \mathbb{C}$, the differential equation

$$
\mathfrak{L}_{\alpha, x} u(x)+\lambda u(x)=0, \quad x \in[-1,0) \cup(0,1]
$$

has a unique solution

$$
\chi(x, \lambda)= \begin{cases}\chi_{1}(x, \lambda), & x \in[-1,0), \\ \chi_{2}(x, \lambda), & x \in(0,1]\end{cases}
$$

satisfying fractional boundary condition (4) and fractional transmission conditions (5) and (6) for each $x \in[-1,0) \cup(0,1]$. 
Remark 2 If $\lambda$ is not eigenvalue, then $\phi_{1}$ and $\chi_{1}$ are linearly independent solutions of equation (2) in the interval $[-1,0)$. Similarly, $\phi_{2}$ and $\chi_{2}$ are linearly independent solutions of equation (2) in the interval $(0,1]$. Then it is obvious that the four functions $\tilde{u_{1}}, \tilde{u_{2}}, \tilde{u}_{3}$, $\tilde{u_{4}}$ which are defined by

$$
\begin{aligned}
& \tilde{u_{1}}=\left\{\begin{array}{ll}
\phi_{1}(x, \lambda), & x \in[-1,0), \\
0, & x \in(0,1],
\end{array} \quad \tilde{u_{2}}= \begin{cases}\chi_{1}(x, \lambda), & x \in[-1,0), \\
0, & x \in(0,1],\end{cases} \right. \\
& \tilde{u_{3}}=\left\{\begin{array}{ll}
0, & x \in[-1,0), \\
\phi_{2}(x, \lambda), & x \in(0,1],
\end{array} \quad \tilde{u}_{4}= \begin{cases}0, & x \in[-1,0), \\
\chi_{2}(x, \lambda), & x \in(0,1]\end{cases} \right.
\end{aligned}
$$

are linearly independent solutions of equation (2) in whole $[-1,0) \cup(0,1]$.

To prove this fact, suppose if possible that $\lambda=\lambda_{0}$ is not an eigenvalue but the corresponding solutions $\phi_{1}\left(x, \lambda_{0}\right)$ and $\chi_{1}\left(x, \lambda_{0}\right)$ are linearly dependent. Then there is a constant $\alpha_{0} \neq 0$ such that

$$
\chi_{1}\left(x, \lambda_{0}\right)=\alpha_{0} \phi_{1}\left(x, \lambda_{0}\right)
$$

From this equality obviously follows that the solution $\chi_{1}\left(x, \lambda_{0}\right)$ also satisfies the first boundary condition. Consequently, the solution

$$
\chi(x, \lambda)= \begin{cases}\chi_{1}\left(x, \lambda_{0}\right), & x \in[-1,0), \\ \chi_{2}\left(x, \lambda_{0}\right), & x \in(0,1]\end{cases}
$$

satisfies also the first boundary condition. Therefore, $\chi\left(x, \lambda_{0}\right)$ satisfies all boundary and transmission conditions, that is, $\chi\left(x, \lambda_{0}\right)$ is an eigenfunction for the considered problem (2)-(6), and consequently $\lambda_{0}$ is an eigenvalue. Thus we have a contradiction which completes the proof.

Let us consider the fractional Wronskians

$$
\begin{aligned}
\omega_{i}(\lambda) & :=W_{F}\left(\phi_{i}(x, \lambda), \chi_{i}(x, \lambda)\right), \quad i=1,2 \\
& :=I_{-1^{+}}^{1-\alpha} \phi_{i}(x, \lambda) D_{0^{+}}^{\alpha} \chi_{i}(x, \lambda)-I_{0^{+}}^{1-\alpha} \chi_{i}(x, \lambda) D_{-1^{+}}^{\alpha} \phi_{i}(x, \lambda)
\end{aligned}
$$

which are independent of $x$ and are entire functions. The short calculation gives

$$
\omega_{1}(\lambda)=\omega_{2}(\lambda)
$$

Now we may introduce to the consideration the characteristic function

$$
\omega(\lambda):=\omega_{1}(\lambda)=\omega_{2}(\lambda)
$$

Lemma 5 The fractional Wronskian $W_{F}$ satisfies the following relation:

$$
W_{F}(\lambda)=-h_{1} \omega^{3}(\lambda),
$$


where

$$
W_{F}(\lambda)=\left|\begin{array}{llll}
L_{1}\left(\phi_{1}\right) & L_{1}\left(\chi_{1}\right) & L_{1}\left(\phi_{2}\right) & L_{1}\left(\chi_{2}\right) \\
L_{2}\left(\phi_{1}\right) & L_{2}\left(\chi_{1}\right) & L_{2}\left(\phi_{2}\right) & L_{2}\left(\chi_{2}\right) \\
L_{3}\left(\phi_{1}\right) & L_{3}\left(\chi_{1}\right) & L_{3}\left(\phi_{2}\right) & L_{3}\left(\chi_{2}\right) \\
L_{4}\left(\phi_{1}\right) & L_{4}\left(\chi_{1}\right) & L_{4}\left(\phi_{2}\right) & L_{4}\left(\chi_{2}\right)
\end{array}\right| .
$$

Proof Employing the definitions of the functions $\phi_{i}(x, \lambda)$ and $\chi_{i}(x, \lambda), i=1,2$, we obtain

$$
\begin{aligned}
W_{F}(\lambda) & =\left|\begin{array}{cccc}
0 & \omega_{1}(\lambda) & 0 & 0 \\
0 & 0 & \omega_{2}(\lambda) & 0 \\
h_{1} I_{-1^{+}}^{1-\alpha} \phi_{1}(-0, \lambda) & h_{1} I_{-1^{+}}^{1-\alpha} \chi_{1}(-0, \lambda) & I_{0^{+}}^{1-\alpha} \phi_{2}(+0, \lambda) & I_{0^{+}}^{1-\alpha} \chi_{2}(+0, \lambda) \\
D_{-1^{+}}^{\alpha} \phi_{1}(-0, \lambda) & D_{-1^{+}}^{\alpha} \chi_{1}(-0, \lambda) & h_{2} D_{0^{+}}^{\alpha} \phi_{2}(+0, \lambda) & h_{2} D_{0^{+}}^{\alpha} \chi_{2}(+0, \lambda)
\end{array}\right| \\
& =\omega_{1}(\lambda) \omega_{2}(\lambda)\left|\begin{array}{cc}
h_{1} I_{-1^{+}}^{1-\alpha} \phi_{1}(-0, \lambda) & I_{0^{+}}^{1-\alpha} \chi_{2}(+0, \lambda) \\
D_{-1^{+}}^{\alpha} \phi_{1}(-0, \lambda) & h_{2} D_{0^{+}}^{\alpha} \chi_{2}(+0, \lambda)
\end{array}\right| \\
& =-h_{1} \omega_{1}^{2}(\lambda) \omega_{2}(\lambda) \\
& =-h_{1} \omega^{3}(\lambda) .
\end{aligned}
$$

Corollary 3 The zeros of the function $W_{F}(\lambda)$ consist of the zeros of the characteristic function $\omega(\lambda)$.

Theorem 4 The eigenvalues of fractional boundary value problem (2)-(6) are the same as the roots of the characteristic function $\omega(\lambda)$.

Proof Let $\lambda=\lambda_{0}$ be a root of the characteristic function $\omega(\lambda)$, hence $\omega_{2}\left(\lambda_{0}\right)=0$. It follows that $\phi_{2}$ and $\chi_{2}$ are linearly dependent, that is,

$$
\phi_{2}\left(x, \lambda_{0}\right)=c \chi_{2}(x, \lambda) ; \quad x \in(0,1]
$$

for some $c \neq 0$. As a result, the function $\phi_{2}\left(x, \lambda_{0}\right)$ satisfies fractional boundary condition (4). So, $\phi\left(x, \lambda_{0}\right)$ which is given by

$$
\phi\left(x, \lambda_{0}\right)= \begin{cases}\phi_{1}\left(x, \lambda_{0}\right), & x \in[-1,0), \\ \phi_{2}\left(x, \lambda_{0}\right), & x \in(0,1]\end{cases}
$$

satisfies the main problem (2)-(6). So, the function $\phi\left(x, \lambda_{0}\right)$ is an eigenfunction of problem (2)-(6) corresponding to the eigenvalue $\lambda_{0}$.

Let $\lambda=\lambda_{0}$ be an eigenvalue and $u_{0}\left(x, \lambda_{0}\right)$ be any corresponding eigenfunction. It must be proved that $\omega\left(\lambda_{0}\right)=0$. Let us suppose that $\omega\left(\lambda_{0}\right) \neq 0$. Then, since $\omega_{1}\left(\lambda_{0}\right) \neq 0$ and $\omega_{2}\left(\lambda_{0}\right) \neq$ 0 , there exist constants $c_{i}, i=1,2,3,4$, at least one of which is not zero, such that

$$
u_{0}\left(x, \lambda_{0}\right)= \begin{cases}c_{1} \phi_{1}\left(x, \lambda_{0}\right)+c_{2} \chi_{1}\left(x, \lambda_{0}\right), & x \in[-1,0), \\ c_{3} \phi_{2}\left(x, \lambda_{0}\right)+c_{4} \chi_{2}\left(x, \lambda_{0}\right), & x \in(0,1]\end{cases}
$$

since $\omega_{1}\left(\lambda_{0}\right) \neq 0$ and $\omega_{2}\left(\lambda_{0}\right) \neq 0$. 
Since the eigenfunction $u_{0}\left(x, \lambda_{0}\right)$ satisfies both fractional boundary and fractional transmission conditions (3)-(6), we have

$$
L_{i} u_{0}\left(\cdot, \lambda_{0}\right)=0, \quad \text { for } i=1,2,3,4 \text {. }
$$

Also since at least one of the constants $c_{i}, i=1,2,3,4$, is not zero,

$$
\operatorname{det}\left(L_{i} u_{0}\left(\cdot, \lambda_{0}\right)\right)=0
$$

that is, $W_{F}(\lambda)=0$. But, by Lemma $5, W_{F}(\lambda) \neq 0$. This contradiction completes the proof.

\section{Acknowledgements}

We are immensely grateful to Prof. Oktay Muhtaroğlu for his expert advice and for sharing his pearls of wisdom with us throughout preparing this research, and we also thank the anonymous reviewers for their valuable comments.

\section{Competing interests}

The authors declare that they have no competing interests.

\section{Authors' contributions}

All authors read and approved the final manuscript.

\section{Publisher's Note}

Springer Nature remains neutral with regard to jurisdictional claims in published maps and institutional affiliations.

Received: 31 July 2017 Accepted: 27 November 2017 Published online: 06 December 2017

\section{References}

1. Podlubny, I: Fractional Differential Equations. Academic Press, San Diego (1999)

2. Kilbass, AA, Srivastava, HM, Trujillo, JJ: Theory and Applications of Fractional Differential Equations. Elsevier, Amsterdam (2006)

3. Baleanu, D, Diethelm, K, Scalas, E, Trujillo, JJ: Fractional Calculus Models and Numerical Methods. Series on Complexity, Nonlinearity and Chaos. World Scientific, Singapore (2012)

4. Yu, Y, Perdikaris, P, Karniadakis, GE: Fractional modeling of viscoelasticity in 3D cerebral arteries and aneurysms. J. Comput. Phys. (2016). doi:10.1016/j.jcp.2016.06.038

5. Sabatier, J, Agrawal, OP, Tenreiro, JA (eds.): Advances in Fractional Calculus: Theoretical Developments and Applications in Physics and Engineering. Springer, Dordrecht (2007)

6. Yang, XJ, Machado, JT: A new fractional operator of variable order: application in the description of anomalous diffusion. Phys. A, Stat. Mech. Appl. 481, 276-283 (2017)

7. Kassim, MD, Furati, KM, Tatar, NE: Nonexistence of global solutions for a fractional differential problem. J. Comput. Appl. Math. 314, 61-68 (2017)

8. Baleanu, D, Golmankhaneh, AK, Golmankhaneh, AK, Nigmatullin, R: Newtonian law with memory. Nonlinear Dyn. 60(1-2), 81-86 (2010)

9. Chen, Q, Debbouche, A, Luo, Z, Wang, J: Impulsive fractional differential equations with Riemann-Liouville derivative and iterative learning control. Chaos Solitons Fractals 102, 111-118 (2017)

10. Yakar, A: Some generalizations of comparison results for fractional differential equations. Comput. Math. Appl. 62(8), 3215-3220 (2011)

11. Yakar, A: Initial time difference quasilinearization for Caputo fractional differential equations. Adv. Differ. Equ. 2012, 92 (2012)

12. Ashyralyev, A: A survey of results in the theory of fractional spaces generated by positive operators. TWMS J. Pure Appl. Math. 6(2), 129-157 (2015)

13. Keyantuo, V, Lizama, C, Warma, M: Existence, regularity and representation of solutions of time fractional diffusion equations. Adv. Differ. Equ. 21(9/10), 837-886 (2016)

14. Fulton, CT: Two-point boundary value problems with eigenvalue parameter contained in the boundary conditions. Proc. R. Soc. Edinb. 77A, 293-308 (1977)

15. Golmankhaneh, A, Khatuni, T, Porghoveh, N, Baleanu, D: Comparison of iterative methods by solving nonlinear Sturm-Liouville, Burgers and Navier-Stokes equations. Open Phys. 10(4), 966-976 (2012)

16. Likov, AV, Mikhailov, Y: The Theory of Heat and Mass Transfer (1963). Qosenergaizdat (Russian)

17. Shkalikov, AA: Boundary value problems for ordinary differential equations with a parameter in boundary conditions. Trydy Sem. Imeny IG Petrowsgo 9, 190-229 (1983) (Russian)

18. Tikhonov, AN, Samarskii, AA: Equations of Mathematical Physics. Pergamon, Oxford (1963)

19. Voitovich, NN, Katsenelbaum, BZ, Sivov, AN: Generalized Method of Eigen-Vibration in the Theory of Diffraction. Nauka, Moskow (1997) (Russian)

20. Mukhtarov, O, Demir, $\mathrm{H}$ : Coerciveness of the discontinuous initial-boundary value problem for parabolic equations. Isr. J. Math. 114, 239-252 (1999) 
21. Aydemir, K, Mukhtarov, O: On the zeros of eigenfunctions of discontinuous Sturm-Liouville problems. J. Comput. Anal. Appl. 23(1), 417-423 (2017)

22. Olğar, H, Mukhtarov, OS: Weak eigenfunctions of two-interval Sturm-Liouville problems together with interaction conditions. J. Math. Phys. 58(4), 042201 (2017)

23. Tunç, E, Mukhtarov, OSH: Fundamental solutions and eigenvalues of one boundary-value problem with transmission conditions. Appl. Math. Comput. 157(2), 347-355 (2004)

24. Titeux, I, Yakubov, Y: Completeness of root functions for thermal conduction in a strip with piecewise continuous coefficients. Math. Models Methods Appl. Sci. 7(7), 1035-1050 (1997)

25. Akdoğan, Z, Demirci, M, Mukhtarov, O: Discontinuous Sturm-Liouville problem with eigenparameter-dependent boundary and transmission conditions. Acta Appl. Math. 86, 329-344 (2005)

26. Demirci, M, Akdoğan, Z, Mukhtarov, O: Asymptotic behavior of eigenvalues and eigenfunctions of one discontinuous boundary-value problem. Int. J. Comput. Cogn. 2(3), 101-113 (2004)

27. Akdoğan, Z, Demirci, M, Mukhtarov, O: Sturm-Liouville problems with eigendependent boundary and transmissions conditions. Acta Math. Sci. 25B(4), 731-740 (2005)

28. Akdoğan, Z, Demirci, M, Mukhtarov, O: Normalized eigenfunctions of discontinuous Sturm-Liouville type problem with transmission conditions. Appl. Math. Sci. 1(52), 2573-2591 (2007)

29. Rivero, M, Trujillo, JJ, Velasco, MP: A fractional approach to the Sturm-Liouville problem. Cent. Eur. J. Phys. 11(10), 1246-1254 (2013)

30. Douglas, R, Anderson, RIA: Fractional-order boundary value problem with Sturm-Liouville boundary conditions. Electron. J. Differ. Equ. 2015(29), 1 (2015)

31. Klimek, M, Agrawal, OP: Fractional Sturm-Liouville problem. Comput. Math. Appl. 66, 795-812 (2013)

32. Zayernouri, M, Karniadakis, GE: Fractional Sturm-Liouville eigen-problems: theory and numerical approximation. J. Comput. Phys. 252, 495-517 (2013)

33. Klimek, M, Odzijewicz, T, Malinowska, AB: Variational methods for the fractional Sturm-Liouville problem. J. Math. Anal. Appl. 416(1), 402-426 (2014)

\section{Submit your manuscript to a SpringerOpen ${ }^{\circ}$ journal and benefit from:}

- Convenient online submission

- Rigorous peer review

- Open access: articles freely available online

- High visibility within the field

- Retaining the copyright to your article

Submit your next manuscript at $\gg$ springeropen.com 